\title{
EFICIÊNCIA E QUALIDADE DA APLICAÇÃO DE MISTURAS EM TANQUE COM ADJUVANTES NA DESSECAÇÃO DE CORDA-DE-VIOLA
}

\section{CLEBER D. DE G. MACIEL ${ }^{1}$, ANTONIO M. DE OLIVEIRA NETO ${ }^{2}$, NAIARA GUERRA ${ }^{2}$, WAGNER JUSTINIANO ${ }^{3}$}

RESUMO: O objetivo do trabalho foi avaliar se a utilização de adjuvantes nas misturas em tanque de glyphosate + chlorimuron-ethyl e glyphosate + carfentrazone-ethyl influenciam na qualidade da aplicação e eficiência de controle de Ipomoea grandifolia. Treze tratamentos, dispostos em delineamento inteiramente casualizado, foram constituídos pelas misturas em tanque de glyphosate + chlorimuron-ethyl $\left(1.440+12,5 \mathrm{~g} \mathrm{ha}^{-1}\right)$ e glyphosate + carfentrazone-ethyl $\left(1.440+10 \mathrm{~g} \mathrm{ha}^{-1}\right)$ isoladas e associados aos adjuvantes Nimbus ${ }^{\circledR}$, Joint Oil ${ }^{\circledR}$, Naturl'Oil ${ }^{\circledR}$, Triunfo $515^{\circledR}$ e All Fix ${ }^{\circledR}$, e uma testemunha sem aplicação. Os adjuvantes utilizados foram eficientes na redução da tensão superficial das caldas; no entanto, não influenciaram nos valores de deposição e na eficiência do controle. Os adjuvantes proporcionaram efeitos distintos na redução da tensão superficial, no $\mathrm{pH}$ e na qualidade da aplicação (diâmetro mediano volumétrico, densidade de gotas, potencial de perdas por deriva, área coberta e diâmetro médio das gotas) das misturas em tanque de glyphosate + chlorimuron-ethyl e glyphosate + carfentrazone-ethyl, mas não interferiram na deposição da calda de pulverização sobre os alvos I. grandifolia e lâminas de vidro. Glyphosate + carfentrazone-ethyl apresentou maior velocidade de controle de I. grandifolia em relação ao glyphosate + chlorimuronethyl, na ausência ou na presença dos adjuvantes, apesar de o glyphosate + carfentrazone-ethyl associado com Nimbus ${ }^{\circledR}$ e Joint $\mathrm{Oil}^{\circledR}$ terem proporcionado às plantas-alvo início de desenvolvimento de novas folhas.

PALAVRAS-CHAVE: tecnologia de aplicação, glyphosate, chlorimuron-ethyl, carfentrazone-ethyl.

\section{EFFICIENCY AND QUALITY OF TANK MIXTURES APPLICATION WITH ADJUVANTS IN MORNINGGLORY DESSECATION}

ABSTRACT: The objective of this study was to evaluate if the use of adjuvants with glyphosate + chlorimuron-ethyl and glyphosate + carfentrazone-ethyl tank mixture influence the quality application and effectiveness for controlling Ipomoea grandifolia. Thirteen treatments, disposed in a completely randomized design, were constituted by tank mixtures of glyphosate + chlorimuronethyl $\left(1440+12,5 \mathrm{~g} \mathrm{ha}^{-1}\right)$ and glyphosate + carfentrazone-ethyl $\left(1440+10 \mathrm{~g} \mathrm{ha}^{-1}\right)$ isolated and associated to Nimbus ${ }^{\circledR}$, Joint $\mathrm{Oil}^{\circledR}{ }^{\circledR}$, Naturl'Oil ${ }^{\circledR}$, Triunfo $515^{\circledR}$ and All Fix ${ }^{\circledR}$, and a control without application. The adjuvants used were efficient in the reduction of the spray superficial tension; however, did not influence deposition values and control efficiency. Adjuvants provided distinct effects in the reduction of superficial tension, $\mathrm{pH}$ and application quality (volumetric median diameter, drops density, potential losses for drift, covered area and drops medium diameter) of glyphosate + chlorimuron-ethyl and glyphosate + carfentrazone-ethyl tank mixtures, but did not interfere in pulverization spray deposition over I. grandifolia targets and glass sheets. Glyphosate + carfentrazone-ethyl presented larger speed for controlling I. grandifolia in relation to glyphosate + chlorimuron-ethyl, in the absence or presence of adjuvants, in spite of glyphosate + carfentrazone-

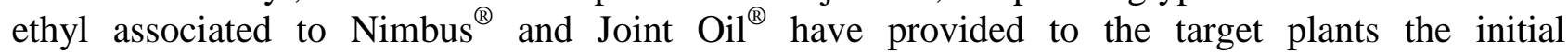
development of new leaves.

KEYWORDS: application technology, glyphosate, chlorimuron-ethyl, carfentrazone-ethyl.

\footnotetext{
${ }^{1}$ Eng ${ }^{\circ}$ Agrônomo., D.Sc., Professor Adjunto, Departamento de Ciências Agronômicas, Universidade Estadual de Maringá - UEM, Câmpus Avançado de Umuarama, Estrada da Paca, s/n, Umuarama, cdgmaciel2@uem.br.

${ }^{2}$ Discentes do Programa de Pós-Graduação em Agronomia, Universidade Estadual de Maringá - UEM/NAPD, Av. Colombo, 5790, Maringá - PR, am.oliveiraneto@hotmail.com; naiara.guerra@hotmail.com.

${ }^{3}$ Discente do Programa em Entomologia e Conservação da Biodiversidade, Universidade Federal da Grande Dourados - UFDG, Rodovia Dourados/Itahum, km 12, Dourados - MS, wanagro@hotmail.com.

Recebido pelo Conselho Editorial em: 5-1-2010

Aprovado pelo Conselho Editorial em: 30-4-2011
} 


\section{INTRODUÇÃO}

O gênero Ipomoea é o que mais se destaca na família Convolvulaceae, uma vez que é constituído por 600 a 700 espécies distribuída no mundo. Dentre as espécies do gênero, existem diversas plantas daninhas que são importantes infestantes de culturas anuais e perenes, destacandose a Ipomoea hederifolia, I. quamoclit, I. purpurea, I. grandifolia e I. nil, denominadas cordas-deviola (KISSMANN \& GROTH, 1999).

Para o controle de Ipomoea spp., o uso do herbicida glyphosate isolado não vem apresentando bons resultados por apresentar translocação diferenciada entre parte aérea e raízes (MONQUERO et al., 2004). LACERDA \& VITÓRIA FILHO (2004) obtiveram níveis de controle inferiores a $80 \%$ na dose de $1.440 \mathrm{~g}$ e.a. ha ${ }^{-1}$ no estádio de três pares de folhas expandidas, apresentando RC 50 (dose necessária para redução de $50 \%$ do desenvolvimento das plantas em relação à testemunha) de 615,49 g e.a. ha ${ }^{-1}$, sendo esta dose inferior somente à obtida para Commelina benghalensis, que apresenta mecanismos de tolerância a este herbicida (MONQUERO et al., 2004; MONQUERO et al., 2005). Portanto, a mistura em tanque entre herbicidas, apesar de ser proibida pelo MAPA, pode ser alternativa eficiente para a solução de tolerância para espécies de plantas daninhas de difícil controle.

A mistura em tanque de glyphosate com herbicidas pode proporcionar outros benefícios além da melhoria na eficiência de plantas daninhas de difícil controle. Alguns herbicidas comumente associados ao glyphosate apresentam atividade residual no solo, permitindo o controle em préemergência e, desta forma, reduzindo a matocompetição inicial (JAREMTCHUCK et al., 2008). A associação de herbicidas com ação residual juntamente com produtos de ação total, como o glyphosate, é uma alternativa para reduzir a infestação de plantas daninhas (CARVALHO et al. 2000; VANLIESHOUT \& LOUX, 2000). O uso da mistura em tanque de glyphosate com chlorimuron-ethyl tem sido considerado uma alternativa vantajosa para o controle de plantas de Bidens pilosa (CARVALHO \& CAVAZZANA, 2000; CARVALHO et al., 2003; ROMAN, 2002) e Euphorbia heterophylla (CARVALHO et al., 2003) em aplicação em pré-semeadura da soja.

Segundo THEISEN et al. (2004), os adjuvantes melhoram o ambiente da calda de pulverização e as condições para a proteção e absorção dos herbicidas, uma vez que boa parte desses produtos dificilmente chegaria ao alvo (sítio enzimático) sem o acréscimo de algum tipo de adjuvante. DURIGAN (1993) menciona que os adjuvantes, quando adicionados à calda de pulverização e que apresentam ação interface, são classificados como surfatantes, e sua utilização prática pode ainda evidenciar efeitos espalhantes, adesionantes e umectantes. A adição de adjuvantes à calda de herbicidas, na maioria das vezes, visa ao aumento da área de cobertura, porém nem sempre maior área de cobertura reflete em maior eficiência biológica (STOCK \& BRIGGS, 2000). STRAHAN et al. (2000) relataram que o uso de adjuvantes não influenciou significativamente na eficiência do herbicida nicosulfuron sobre Rottboellia cochinchinensis.

O objetivo do presente trabalho foi avaliar se a utilização de adjuvantes nas misturas em tanque de glyphosate + chlorimuron-ethyl e glyphosate + carfentrazone-ethyl influencia na qualidade da aplicação e na eficácia de controle de Ipomoea grandifolia.

\section{MATERIAL E MÉTODOS}

O experimento foi conduzido em estufa plástica, nas dependências do Câmpus da Escola Superior de Agronomia de Paraguaçu Paulista - ESAPP, município de Paraguaçu Paulista-SP, localizado em altitude média de $506 \mathrm{~m}$ e latitude de $22^{\circ} 34^{\prime} 53^{\prime \prime} \mathrm{S}$ e longitude de $50^{\circ} 34^{\prime} 35^{\prime \prime}$ W, região pertencente ao Vale do Paranapanema. A amostra de solo utilizada no experimento foi retirada da camada superficial de $0-10 \mathrm{~cm}$ e peneirada em peneira de $2 \mathrm{mesh}$, com a exclusão de raízes e torrões. A análise física de solo indicou ser ele classificado como textura arenosa, por constituir-se de $80,3 \%$ de areia; $12,2 \%$ de argila, 7,5 \% de silte, e a análise química com pH de 5,5

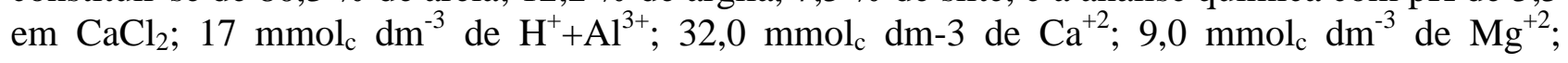


1,3 mmolc $\mathrm{dm}^{-3}$ de $\mathrm{K}^{+}$; 32,0 $\mathrm{mg} \mathrm{dm}^{-3}$ de P; 12,0 $\mathrm{g} \mathrm{dm}^{-3}$ de MO; SB de 42,0 $\mathrm{mmol}_{\mathrm{c}} \mathrm{dm}^{-3}$; CTC de $59,0 \mathrm{mmol}_{\mathrm{c}} \mathrm{dm}^{-3}$ e V\% de $71,0 \%$.

As unidades experimentais foram constituídas por vasos com capacidade de $10 \mathrm{~kg}$ de solo, onde foram semeadas 10 sementes de I. grandifolia por vaso, no dia 28-6-2008. No momento da aplicação dos tratamentos, a maioria das plantas daninhas estava no final do estádio vegetativo, e algumas plantas iniciavam a emissão das estruturas reprodutivas.

Os tratamentos foram constituídos pela mistura em tanque de glyphosate + chlorimuron-ethyl $\left(1440+12,5 \mathrm{~g} \mathrm{ha}^{-1}\right)$ e glyphosate + carfentrazone-ethyl $\left(1440+10 \mathrm{~g} \mathrm{ha}^{-1}\right)$ isoladas e associadas aos adjuvantes Nimbus ${ }^{\circledR}$, Joint Oil ${ }^{\circledR}$, Naturl'Oil ${ }^{\circledR}$, Triunfo $515^{\circledR}$ e All Fix ${ }^{\circledR}$, todos a $0,2 \% \mathrm{v} \mathrm{v}^{-1}$, e uma testemunha sem aplicação, totalizando 13 tratamentos com cinco repetições, dispostos em delineamento inteiramente casualizado. Na calda de pulverização de todos os tratamentos, foram adicionados 1.500 ppm do corante alimentício azul brilhante FD\&C-1, com objetivo de utilizá-lo como traçador da solução, visando ao monitoramento da deposição das caldas de pulverização. Na superfície do solo das unidades experimentais, foi posicionada uma lamina de vidro $(2,6 \times 7,6 \mathrm{~cm}) \mathrm{e}$ três papéis hidrossensíveis $(2,6 \times 7,6 \mathrm{~cm})$, para a avaliação dos parâmetros da qualidade da pulverização.

A aplicação foi realizada no dia 27-9-2008, utilizando pulverizador costal pressurizado a $\mathrm{CO}_{2}$, com duas pontas DG $110.02 \mathrm{VS}$, espaçadas em $0,5 \mathrm{~m}$ de altura do alvo biológico, com pressão de trabalho a $207 \mathrm{kPa}$, o qual possibilitou uma taxa de aplicação de $150 \mathrm{~L} \mathrm{ha}^{-1}$. No momento da aplicação, o solo encontrava-se úmido, a temperatura do ar entre $25-28{ }^{\circ} \mathrm{C}$, umidade relativa de $55 \%$ e ventos de aproximadamente $3,6 \mathrm{~m} \mathrm{~s}^{-1}$, céu claro e sem a presença de nuvens. Os vasos foram irrigados no dia seguinte, após a aplicação, e posteriormente uma vez ao dia, com o objetivo de apenas manter a umidade do solo a $80 \%$ da capacidade de campo.

Após a aplicação dos tratamentos, foram coletadas as lâminas e três folhas das plantas de $I$. grandifolia por unidade experimental, nas cinco repetições. Na sequência, esses materiais foram embalados separadamente em sacos plásticos e encaminhados ao laboratório para procedimentos de lavagem do material com $25 \mathrm{~cm}^{-3}$ de água destilada, através de agitação manual e constante por 20 segundos, para a retirada do traçador dos referidos alvos. Após a lavagem das amostras, realizou-se a determinação da área foliar da I. grandifolia através de um integrador de área foliar tipo Areameter, modelo Licor $3100^{\circledR}$. A determinação da quantidade de traçante depositado foi efetuada utilizando espectrofotômetro, cujos resultados da leitura em absorbância no comprimento de onda de $630 \mathrm{~nm}$ proporcionaram a transformação em $\mathrm{mg} \mathrm{L}^{-1}$, de acordo com o coeficiente angular da curva-padrão. Os valores de depósitos foram posteriormente transformados em $\mu \mathrm{L} \mathrm{cm}{ }^{-2}$, conforme metodologia proposta por MACIEL et al. (2007).

Os papéis hidrossensíveis também foram cuidadosamente coletados após a aplicação e submetidos à digitalização em scanner para a avaliação dos parâmetros da pulverização, conforme recomendação do software e-Sprinkle ${ }^{\circledR}$ (EMBRAPA, 2000), de forma semelhante à descrita por GARCIA et al. (2004). Nessa etapa, foram avaliados os parâmetros diâmetro mediano volumétrico (DMV), densidade de gotas por $\mathrm{cm}^{2}$, potencial de perdas por deriva (\%PDR), área coberta pelas gotas (\%AC) e diâmetro médio das gotas.

As tensões superficiais estáticas das soluções foram avaliadas segundo metodologia descrita por MACIEL et al. (2010), medindo-se a massa das gotas formadas na extremidade de uma bureta posicionada dentro de uma balança analítica (precisão de $0,01 \mathrm{mg}$ ), em tempo determinado entre 25 e 30 segundos. Cada conjunto de três gotas pesadas correspondeu a uma repetição, em que, para evitar perdas por evaporação, utilizou-se para captura das gotas óleo vegetal em bécker de $25 \mathrm{~cm}^{-3}$, posicionado abaixo da ponta da bureta e sobre o prato da balança, totalizando vinte repetições amostradas por tratamento.

A variável-resposta percentual de controle foi avaliada aos $3 ; 7 ; 14$ e 21 dias após a aplicação dos tratamentos (DAA), de acordo com recomendações da Sociedade Brasileira da Ciência das 
Plantas Daninhas (SBCPD, 1995), em que onde o valor 0 representa a ausência de controle (testemunha sem aplicação), e 100, a morte das plantas; considerando que controle igual ou superior a $80 \%$ foi considerado satisfatório. Aos 21 DAA, foi coletada a parte aérea das plantas de $I$. grandifolia, embaladas em sacos de papel e acondicionadas em estufa de circulação de ar forçada a $65^{\circ} \mathrm{C}$ até atingir massa constante, sendo, posteriormente, quantificada a massa seca da parte aérea em balança digital de precisão de $0,01 \mathrm{~g}$.

Determinou-se a comparação dos parâmetros da aplicação: deposição da pulverização, tensão superficial das soluções, diâmetro mediano volumétrico, densidade de gotas por $\mathrm{cm}^{2}$, potencial de perdas por deriva, área coberta pelas gotas, diâmetro médio das gotas e controle das plantas daninhas, através de três contrastes, conforme metodologia descrita por BANZATTO \& KRONKA (2008). Essa comparação teve por finalidade analisar de maneira global o comportamento dos dois grupos de mistura em tanque de herbicidas sobre estas variáveis-resposta em todos os tratamentos (Contraste 1), assim como verificar se o uso dos adjuvantes favoreceu o desempenho da mistura de glyphosate + chlorimuron-ethyl (Contraste 2) e glyphosate + carfentrazone-ethyl (Contraste 3 ). Os contrastes utilizados estão descritos abaixo:

$$
\begin{aligned}
& \mathrm{C} 1=\mu \mathrm{t} 1+\mu \mathrm{t} 2+\mu \mathrm{t} 3+\mu \mathrm{t} 4+\mu \mathrm{t} 5+\mu \mathrm{t} 6-\mu \mathrm{t} 7-\mu \mathrm{t} 8-\mu \mathrm{t} 9-\mu \mathrm{t} 10-\mu \mathrm{t} 11-\mu \mathrm{t} 12 \\
& \mathrm{C} 2=5 \mu \mathrm{t} 1-\mu \mathrm{t} 2-\mu \mathrm{t} 3-\mu \mathrm{t} 4-\mu \mathrm{t} 5-\mu \mathrm{t} 6 \\
& \mathrm{C} 3=5 \mu \mathrm{t} 7-\mu \mathrm{t} 8-\mu \mathrm{t} 9-\mu \mathrm{t} 10-\mu \mathrm{t} 11-\mu \mathrm{t} 12
\end{aligned}
$$

em que,

$\mu \mathrm{t}$ - media dos tratamentos envolvidos nos contrastes.

De forma similar aos outros parâmetros, realizou-se a análise por contraste para a variávelresposta matéria seca da parte aérea; porém, neste caso, o contraste 1 consistiu na comparação entre a produção de massa seca da testemunha com os tratamentos, e os demais contrastes foram idênticos aos citados acima (Contrastes 2; 3 e 4), a significância das estimativas obtidas em cada contraste foi testada por "F"a 5\% de probabilidade de erro. Os contrastes utilizados para a variávelresposta matéria seca da parte aérea estão descritos abaixo.

$$
\begin{aligned}
& \mathrm{C} 1=12 \mu \mathrm{t}-\mu \mathrm{t} 1-\mu \mathrm{t} 2-\mu \mathrm{t} 3-\mu \mathrm{t} 4-\mu \mathrm{t} 5-\mu \mathrm{t} 6-\mu \mathrm{t} 7-\mu \mathrm{t} 8-\mu \mathrm{t} 9-\mu \mathrm{t} 10-\mu \mathrm{t} 11-\mu \mathrm{t} 12 \\
& \mathrm{C} 2=\mu \mathrm{t} 1+\mu \mathrm{t} 2+\mu \mathrm{t} 3+\mu \mathrm{t} 4+\mu \mathrm{t} 5+\mu \mathrm{t} 6-\mu \mathrm{t} 7-\mu \mathrm{t} 8-\mu \mathrm{t} 9-\mu \mathrm{t} 10-\mu \mathrm{t} 11-\mu \mathrm{t} 12 \\
& \mathrm{C} 3=5 \mu \mathrm{t} 1-\mu \mathrm{t} 2-\mu \mathrm{t} 3-\mu \mathrm{t} 4-\mu \mathrm{t} 5-\mu \mathrm{t} 6 \\
& \mathrm{C} 4=5 \mu \mathrm{t} 7-\mu \mathrm{t} 8-\mu \mathrm{t} 9-\mu \mathrm{t} 10-\mu \mathrm{t} 11-\mu \mathrm{t} 12
\end{aligned}
$$

em que,

$\mu \mathrm{t}$ - média dos tratamentos envolvidos nos contrastes.

A significância das estimativas obtidas em cada contraste foi testada por " $F$ " a $5 \%$ de probabilidade de erro. Os dados obtidos foram submetidos à análise de variância pelo teste $\mathrm{F}$, e as suas médias, comparadas pelo teste de agrupamento de médias de Scott-Knott (SCOTT \& KNOTT, 1974), a 5\% de probabilidade de erro, com a finalidade de avaliar o efeito individual de cada tratamento, em cada variável-resposta avaliada.

\section{RESULTADOS E DISCUSSÃO}

O uso de adjuvantes não influenciou na deposição da calda de pulverização das misturas de glyphosate com chlorimuron-ethyl ou carfentrazone-ethyl sobre as folhas de I. grandifolia e lâminas posicionadas na superfície do solo (Tabela 1). Os resultados dos contrastes estudados indicam que a deposição da pulverização do grupo de tratamentos com glyphosate + chlorimuron-ethyl não diferiu do grupo com glyphosate + carfentrazone-ethyl, assim como a adição de adjuvantes não influenciou na deposição sobre as folhas da planta daninha e lâminas de vidro (Figura 1). Nesse sentido, os resultados corroboram os descritos por SMITH (2000) e MACIEL et al. (2001), em que não foram 
encontradas alterações significativas na deposição da pulverização em folhas de Cassia occidentalis e I. grandifolia, respectivamente, com ou sem a adição de adjuvantes. Para MILLER (1993), alvos naturais, apesar de constituírem fielmente as características do alvo estudado, apresentam complexibilidade e variabilidade natural que afetam a retenção e o espalhamento do produto aplicado.

TABELA1. Análise dos contrastes entre tratamentos para depósitos das caldas de pulverização das misturas glyphosate + chlorimuron-ethyl e glyphosate + carfentrazone-ethyl, isolados e associados a adjuvantes sobre folhas de Ipomoea grandifolia e lâminas de vidro, posicionadas na superfície do solo. Contrasts analysis among treatments for spraying volume deposits of glyphosate + chlorimuron-ethyl and glyphosate + carfentrazone-ethyl mixtures isolated and associated with adjuvants over Ipomoea grandifolia leaves and sheets of glass, positioned on soil surface.

\begin{tabular}{lccc}
\hline \multicolumn{1}{c}{ Folhas } & & \\
\hline \multicolumn{1}{c}{ Contrastes } & Estimativas & Fc & Pr>Fc \\
\hline Contraste 1 (glyphosate + chlor ${ }^{1}$ X glyphosate + carf ${ }^{2}$ ) & 0,12392593 & $3,867^{\mathrm{ns}}$ & 0,0551 \\
Contraste 2 (glyphosate + chlor X glyphosate + chlor + adjuvantes) & 0,16280000 & $1,854^{\mathrm{ns}}$ & 0,1797 \\
Contraste 3 (glyphosate + carf X glyphosate + carf + adjuvantes) & 0,04271111 & $0,128^{\mathrm{ns}}$ & 0,7225 \\
\hline \multicolumn{1}{c}{ Lâminas } & & \\
\hline Contraste 1 (glyphosate + chlor X glyphosate + carf) & 0,04629630 & $0,152^{\mathrm{ns}}$ & 0,6983 \\
Contraste 2 (glyphosate + chlor X glyphosate + chlor + adjuvantes) & 0,32520000 & $2,085^{\mathrm{ns}}$ & 0,1553 \\
Contraste 3 (glyphosate + carf X glyphosate + carf + adjuvantes) & 0,00395556 & $0,000^{\mathrm{ns}}$ & 0,9861 \\
\hline
\end{tabular}

Obs.: estimativas $=$ estimativa do valor dos contrastes $1 ; 2$ e $3 . \mathrm{Fc}=\mathrm{F}$ calculado $; \mathrm{Pr}>\mathrm{Fc}=$ probabilidade de aceitar ou não a hipótese como verdadeira. ${ }^{1}$ chlorimuron-ethyl; ${ }^{2}$ carfentrazone-ethyl. ${ }^{\text {ns }}$ não significativo pelo teste $\mathrm{F}$, a $5 \%$ de probabilidade.

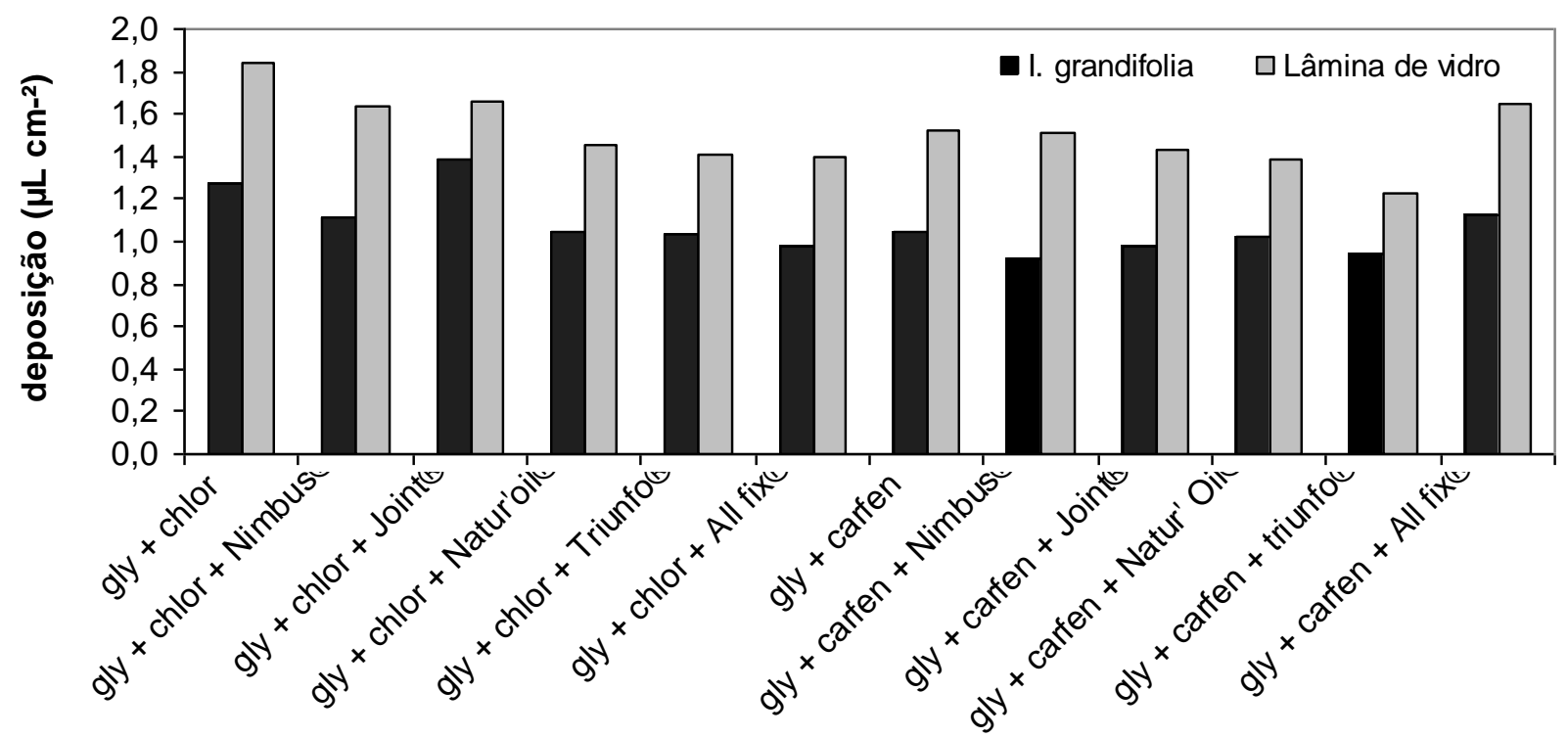

FIGURA 1. Deposição média da calda de pulverização das misturas glyphosate + chlorimuron-ethyl (gly+chlor) e glyphosate + carfentrazone-ethyl (gly+carfen), isolados e associados a adjuvantes sobre os alvos folhas de Ipomoea grandifolia e lâminas de vidro, posicionadas na superfície do solo. Spraying volume medium deposition of glyphosate + chlorimuron-ethyl (gly+chlor) and glyphosate + carfentrazone-ethyl (gly+carfen) mixtures isolated and associated to adjuvants over Ipomoea grandifolia leaves targets and sheets of glass, positioned on soil surface.

O valor da estimativa do contraste 1 para as médias de tensão superficial (Tabela 2) foi positivo e significativo $(\mathrm{p}<0,05)$, indicando que os valores médios da tensão superficial foram 
maiores para misturas com glyphosate + chlorimuron-ethyl, quando comparados ao glyphosate + carfentrazone-ethyl. Para os resultados do contrastes 2 e 3, nota-se que o uso dos adjuvantes favoreceu significativamente na redução da tensão superficial da calda de pulverização. A submissão dos dados ao teste de agrupamento de Scott-Knott demonstrou que, em média, a maioria dos adjuvantes foram eficientes na redução da tensão superficial estática das misturas, uma vez que o teste analisa as médias individuais de cada adjuvante (Figura 2). $\mathrm{O}$ adjuvante Nimbus ${ }^{\circledR}$ foi o mais eficiente na redução da tensão superficial das misturas em tanque de glyphosate com chlorimuronethyl e com carfentrazone-ethyl, obtendo valores médios de tensão de 31,7 e $33,0 \mathrm{mN} \mathrm{m}^{-1}$, respectivamente. MACIEL et al. (2010) também relataram que o Nimbus ${ }^{\circledR}$ se mostrou eficiente na redução da tensão superficial para mistura em tanque das formulações de glyphosate Roundup ready ${ }^{\circledR}$ e Polaris ${ }^{\circledR}$ com chlorimuron-ethyl.

TABELA 2. Análise dos contrastes entre tratamentos para a tensão superficial das misturas entre glyphosate + chlorimuron-ethyl e glyphosate + carfentrazone-ethyl, isolados e associados a adjuvantes. Contrasts analysis among treatments for superficial tension between glyphosate + chlorimuron-ethyl and glyphosate + carfentrazoneethyl mixtures isolated and associated to adjuvants.

\begin{tabular}{|c|c|c|c|}
\hline Contrastes & Estimativas & $\mathrm{Fc}$ & $\operatorname{Pr}>\mathrm{Fc}$ \\
\hline Contraste 1 (glyphosate + chlor $^{1} \mathrm{X}$ glyphosate + carf $\left.^{2}\right)$ & 0,57500000 & $52,704 *$ & 0,0000 \\
\hline Contraste 2 (glyphosate + chlor X glyphosate + chlor + adjuvantes $)$ & 5,68000000 & $1.428,583 *$ & 0,0000 \\
\hline Contraste 3 (glyphosate + carfen X glyphosate + carfen + adjuvantes) & 1,87000000 & $154,843 *$ & 0,0000 \\
\hline
\end{tabular}

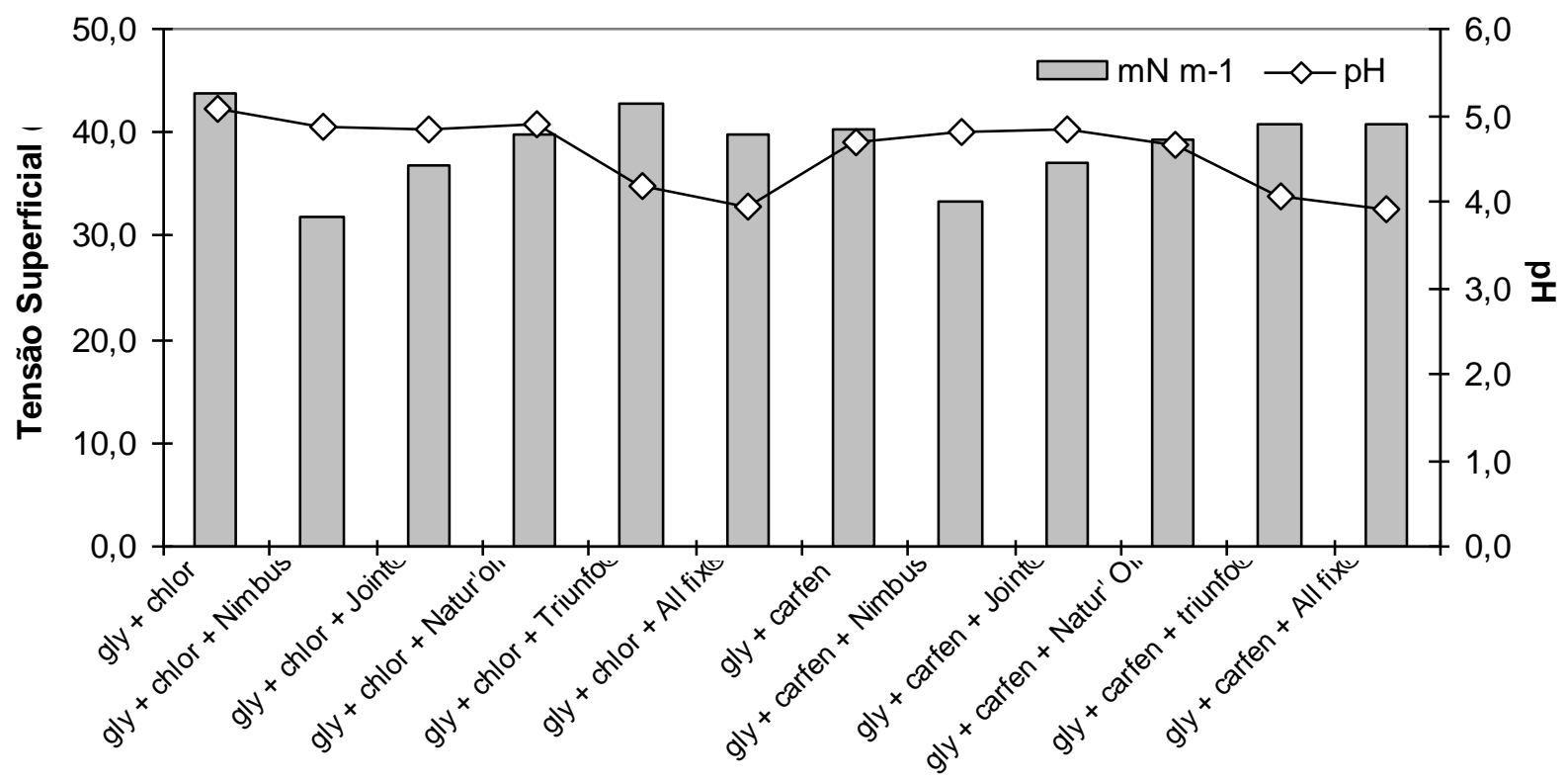

FIGURA 2. Valores de tensão superficial estática e $\mathrm{pH}$ das misturas entre glyphosate + chlorimuron-ethyl (gly+chlor) e glyphosate + carfentrazone-ethyl (gly+carfen), isolados e associados a adjuvantes. Static superficial tension and $\mathbf{p H}$ values of mixtures between glyphosate + chlorimuron-ethyl (gly+chlor) and glyphosate + carfentrazone-ethyl (gly+carfen) isolated and associated to adjuvants.

$\mathrm{O}$ adjuvante Joint $\mathrm{Oil}^{\circledR}$ apresentou intermediário potencial em atuar como tenso ativo, ao contrário do Natur'Oil ${ }^{\circledR}$, All Fix ${ }^{\circledR}$ e Triunfo $515^{\circledR}$ que apresentaram as menores reduções da tensão superficial das misturas glyphosate + chlorimuron-ethyl e glyphosate + carfentrazone-ethyl. 
Quanto à qualidade da aplicação, avaliada por meio de papéis hidrossensíveis, as soluções de glyphosate + chlorimuron-ethyl (contraste 1), associadas aos adjuvantes, apresentaram maiores diâmetros mediano volumétrico (DMV) e médio das gotas, quando comparados ao agrupamento de tratamentos com glyphosate + carfentrazone-ethyl (contraste 2) (Tabela 3). Em contrapartida, o agrupamento envolvendo a mistura de glyphosate + carfentrazone-ethyl apresentou as maiores variáveis-resposta para densidades de gotas $\left(\mathrm{cm}^{2}\right)$, áreas de cobertura $(\%)$ e potencial de perda por deriva (\%). IOST (2008), avaliando o efeito de adjuvantes com potencial antideriva (Antideriva, Uno, Pronto 3, Li-700 e Supersil) no tamanho de gotas de pulverização, notou apenas pequena influência no DMV e potencial de perda por deriva, para as doses recomendadas pelos fabricantes.

O uso de adjuvantes à calda de pulverização de glyphosate + chlorimuron-ethyl proporcionou maior DMV, \% de área coberta e diâmetro médio de gotas, e menor densidade de gotas e PDR (\%). Já quando adicionado á mistura de carfentrazone-ethyl, notou-se maior densidade de gotas e maior \% de área coberta, e para as demais variáveis analisadas não foram obtidas diferenças significativa entre os contrastes analisados (Tabela 3).

TABELA 3. Análise dos contrastes entre tratamentos para diâmetro mediano volumétrico (DMV), densidade de gotas $\left(\mathrm{cm}^{2}\right)$, potencial de perdas por deriva (\%PDR), percentual de área coberta e diâmetro médio das gotas de misturas entre glyphosate + chlorimuron-ethyl e glyphosate + carfentrazone-ethyl, isolados e associados a adjuvantes. Contrasts analysis among treatments for volumetric median diameter (VMD), drops density $\left(\mathrm{cm}^{2}\right)$, potential losses for drift (\% LPD), percentage of covered area and drop medium diameter of mixtures between glyphosate + chlorimuron-ethyl and glyphosate + carfentrazone-ethyl isolated and associated to adjuvants. .

\begin{tabular}{|c|c|c|c|}
\hline \multicolumn{4}{|l|}{ DMV } \\
\hline Contrastes & Estimativas & Fc & $\mathrm{Pr}>\mathrm{Fc}$ \\
\hline Contraste 1 (glyphosate + chlor $^{1} \mathrm{X}$ glyphosate + carf $\left.^{2}\right)$ & 214,96666 & $86,400 *$ & 0,0000 \\
\hline Contraste 2 (glyphosate + chlor X glyphosate + chlor + adjuvantes) & $-5,40000$ & $0,015^{\mathrm{ns}}$ & 0,9031 \\
\hline Contraste 3 (glyphosate + carfen X glyphosate + carfen + adjuvantes) & 203,36000 & $21,478 *$ & 0,0001 \\
\hline \multicolumn{4}{|l|}{ Densidade de Gotas $\left(\mathrm{cm}^{2}\right)$} \\
\hline Contraste 1 (glyphosate + chlor $^{1} \mathrm{X}$ glyphosate + carf $\left.^{2}\right)$ & $-11,38888$ & $7,795^{*}$ & 0,0101 \\
\hline Contraste 2 (glyphosate + chlor X glyphosate + chlor + adjuvantes) & 17,40000 & $5,054 *$ & 0,0340 \\
\hline Contraste 3 (glyphosate + carfen X glyphosate + carfen + adjuvantes) & $-11,46666$ & $2,195^{\mathrm{ns}}$ & 0,1515 \\
\hline \multicolumn{4}{|l|}{ PDR $(\%)$} \\
\hline$\overline{\text { Contraste } 1\left(\text { glyphosate }+ \text { chlor }^{1} \mathrm{X} \text { glyphosate }+ \text { carf }^{2}\right)}$ & $-0,31222$ & $17,118^{*}$ & 0,0004 \\
\hline Contraste 2 (glyphosate + chlor X glyphosate + chlor + adjuvantes $)$ & $-0,01333$ & $0,009^{\mathrm{ns}}$ & 0,9266 \\
\hline Contraste 3 (glyphosate + carfen X glyphosate + carfen + adjuvantes) & $-0,30800$ & $4,627 *$ & 0,0417 \\
\hline \multicolumn{4}{|l|}{ Área Coberta $(\%)$} \\
\hline$\overline{\text { Contraste } 1\left(\text { glyphosate }+ \text { chlor }^{1} \mathrm{X} \text { glyphosate }+ \text { carf }^{2}\right)}$ & 9,44666 & $33,492 *$ & 0,0000 \\
\hline Contraste 2 (glyphosate + chlor X glyphosate + chlor + adjuvantes $)$ & 11,77333 & $14,450 *$ & 0,0009 \\
\hline Contraste 3 (glyphosate + carfen X glyphosate + carfen + adjuvantes) & 4,30933 & $1,936^{\mathrm{ns}}$ & 0,1769 \\
\hline \multicolumn{4}{|l|}{ Diâmetro Médio de Gotas } \\
\hline Contraste 1 (glyphosate + chlor $^{1} \mathrm{X}$ glyphosate + carf $^{2}$ ) & 27,08888 & $14,099 *$ & 0,0010 \\
\hline Contraste 2 (glyphosate + chlor X glyphosate + chlor + adjuvantes $)$ & 9,00000 & $0,4320^{\mathrm{ns}}$ & 0,5171 \\
\hline Contraste 3 (glyphosate + carfen X glyphosate + carfen + adjuvantes) & 16,70666 & $1,49^{\mathrm{ns}}$ & 0,2341 \\
\hline
\end{tabular}


Os adjuvantes Nimbus ${ }^{\circledR}$ e Natur'Oil ${ }^{\circledR}$, quando adicionados à mistura de glyphosate + chlorimuron-ethyl, aumentaram o DMV. Já para glyphosate + carfentrazone-ethyl, todos os adjuvantes testados reduziram esta variável. A densidade de gotas da mistura de glyphosate + chlorimuron-ethyl foi reduzida com o uso dos adjuvantes testados. No entanto, para a mistura de glyphosate + carfentrazone-ethyl, com exceção do Natur'Oil ${ }^{\circledR}$, os demais adjuvantes testados aumentaram a densidade de gotas (Tabela 4).

A adição de adjuvantes não interferiu no potencial de perdas por deriva para o glyphosate + chlorimuron-ethyl; porém, na mistura de glyphosate + carfentrazone-ethyl, os adjuvantes Nimbus ${ }^{\circledR}$, Joint Oil ${ }^{\circledR}$, Triunfo $515^{\circledR}$ e All Fix ${ }^{\circledR}$ aumentaram o potencial de perdas por deriva. A maior área de cobertura foi obtida quando se adicionaram Joint ${ }^{\circledR}$, Triunfo $515^{\circledR}$ e All Fix ${ }^{\circledR}$ à mistura de glyphosate + chlorimuron-ethyl. Já na mistura de glyphosate + carfentrazone-ethyl, não houve interferência da adição de adjuvantes. O diâmetro médio de gotas da mistura de glyphosate + clorimuron-ethyl foi reduzido quando se fez o uso do All Fix ${ }^{\circledR}$. Na mistura de glyphosate + carfentrazone-ethyl, somente o Natur'Oil ${ }^{\circledR}$ não proporcionou redução no diâmetro médio das gotas (Tabela 4).

TABELA 4. Valores de diâmetro mediano volumétrico (DMV), densidade de gotas por $\mathrm{cm}^{2}$ (DEN), percentual do potencial de perdas por deriva (PDR\%), percentual de área coberta $(\mathrm{AC} \%)$ e diâmetro médio de gotas (DIA) em papéis hidrossensíveis. Values of volumetric median diameter (VMD), drop density per $\mathrm{cm}^{2}$ (DEN), percentage of potential losses for drift (LPD\%), percentage of covered area (CA\%) and drop medium diameter (DMD) in hydro sensible papers.

\begin{tabular}{|c|c|c|c|c|c|}
\hline Tratamentos & $\operatorname{DMV}(\mu \mathrm{m})$ & $\operatorname{DEN}\left(\mathrm{cm}^{2}\right)$ & PDR $(\%)$ & $\mathrm{AC}(\%)$ & DIA $(\mu \mathrm{m})$ \\
\hline 1. gly $^{1}+$ chlor $^{2}$ & $1.077 \mathrm{~b}$ & $120 \mathrm{a}$ & $0,40 \mathrm{~b}$ & $62,0 \mathrm{a}$ & $307 \mathrm{a}$ \\
\hline 2. gly + chlor + Nimbus ${ }^{\circledR}$ & $1.194 \mathrm{a}$ & $112 \mathrm{~b}$ & $0,37 \mathrm{~b}$ & $56,1 \mathrm{a}$ & $310 \mathrm{a}$ \\
\hline 3. gly + chlor + Joint Oil $^{\circledR}$ & $1.018 \mathrm{~b}$ & $110 \mathrm{~b}$ & $0,43 \mathrm{~b}$ & $49,4 \mathrm{~b}$ & $300 \mathrm{a}$ \\
\hline 4. gly $^{2}$ chlor + Natur'Oil $^{\circledR}$ & $1.185 \mathrm{a}$ & $97 \mathrm{~b}$ & $0,27 \mathrm{~b}$ & $57,5 \mathrm{a}$ & $312 \mathrm{a}$ \\
\hline 5. gly + chlor + Triunfo $515^{\circledR}$ & $1.039 \mathrm{~b}$ & $92 \mathrm{~b}$ & $0,43 \mathrm{~b}$ & $44,8 \mathrm{~b}$ & $289 \mathrm{a}$ \\
\hline 6. gly + chlor + All Fix ${ }^{\circledR}$ & $976 \mathrm{~b}$ & $100 \mathrm{~b}$ & $0,57 \mathrm{~b}$ & $43,2 \mathrm{~b}$ & $277 \mathrm{~b}$ \\
\hline 7. gly + carfen $^{3}$ & $1.036 \mathrm{~b}$ & $107 \mathrm{~b}$ & $0,47 \mathrm{~b}$ & $46,3 \mathrm{~b}$ & $286 a$ \\
\hline 8. gly + carfen + Nimbus ${ }^{\circledR}$ & $774 \mathrm{c}$ & $125 \mathrm{a}$ & $0,70 \mathrm{a}$ & $44,5 \mathrm{~b}$ & $273 b$ \\
\hline 9. gly + carfen + Joint Oil ${ }^{\circledR}$ & $821 \mathrm{c}$ & $115 \mathrm{a}$ & $0,87 \mathrm{a}$ & $36,0 \mathrm{~b}$ & $262 \mathrm{~b}$ \\
\hline 10. gly + carfen + Natur'Oil ${ }^{\circledR}$ & $915 \mathrm{c}$ & $88 \mathrm{~b}$ & $0,37 \mathrm{~b}$ & $43,5 \mathrm{~b}$ & $309 a$ \\
\hline 11. gly + carfen + Triunfo $515^{\circledR}$ & $804 \mathrm{c}$ & $137 \mathrm{a}$ & $1,14 \mathrm{a}$ & $42,4 \mathrm{~b}$ & $241 \mathrm{~b}$ \\
\hline 12. gly + carfen + All Fix ${ }^{\circledR}$ & $848 \mathrm{c}$ & $127 \mathrm{a}$ & $0,80 \mathrm{a}$ & $43,6 \mathrm{~b}$ & $262 \mathrm{~b}$ \\
\hline$\overline{\mathrm{Pr}>\mathrm{F}}$ & 0,0000 & 0,0003 & 0,0000 & 0,0000 & 0,0009 \\
\hline $\mathrm{Fc}$ & $14,989 *$ & $5,552 *$ & $5,504 *$ & $7,982 *$ & $4,478 *$ \\
\hline
\end{tabular}

Obs. : $\mathrm{Fc}=\mathrm{F}$ calculado; $\mathrm{Pr}>\mathrm{Fc}=$ probabilidade de aceitar ou não a hipótese como verdadeira. ${ }^{1}$ chlorimuron-ethyl; ${ }^{2}$ carfentrazoneethyl; *significativo a $5 \%$ de probabilidade $(\mathrm{p}<0,05)$. Médias seguidas de mesma letra não diferem entre si, a $5 \%$ de probabilidade, pelo teste de Scott-Knot.

O grupo dos tratamentos de glyphosate + carfentrazone-ethyl apresentou controle superior aos tratamentos com glyphosate + chlorimuron-ethyl na avaliação de 21 DAA (Tabela 5). Segundo ROCHA et al. (2007), aos 3 DAA, a mistura em tanque de glyphosate + carfentrazone-ethyl proporcionou controle de $94,2 \%$ e $86,2 \%$ para Commelina benghalensis e $C$. villosa, respectivamente, demonstrando que a associação entre esses herbicidas caracterizou sinergismo para dessecação das plantas daninhas.

Os adjuvantes não influenciaram na eficiência de controle de I. grandifolia para a mistura glyphosate + chlorimuron-ethyl, uma vez que, aos 21 DAA, o resultado da estimativa para o contraste 2 não apresentou diferença significativa pelo teste F. Dessa forma, o uso de adjuvantes não proporcionou benefícios para a variável-resposta eficiência de controle quando associados ao glyphosate + chlorimuron-ethyl (Tabela 5). A testemunha apresentou acúmulo de matéria seca 
superior às plantas tratadas, já que o resultado do primeiro contraste foi positivo e significativo, com incremento de matéria seca média estimada de 3,35 g. Resultados semelhantes ocorreram para o segundo contraste, em que os tratamentos com glyphosate + chlorimuron-ethyl apresentaram quantidade de matéria seca superior aos tratamentos com glyphosate + carfentrazone-ethyl.

TABELA 5. Análise dos contrastes entre tratamentos para eficiência de controle (\%) e matéria seca da parte aérea (g), aos 21 DAA, para misturas entre glyphosate + chlorimuron-ethyl e glyphosate + carfentrazone-ethyl, isolados e associados a adjuvantes. Contrasts analysis among treatments to control efficiency $(\%)$ and dossel dry matter (g) at 21 DAA for mixtures between glyphosate + chlorimuron-ethyl and glyphosate + carfentrazone-ethyl isolated and associated to adjuvants.

\begin{tabular}{|c|c|c|c|}
\hline \multicolumn{4}{|l|}{ Eficiência de Controle } \\
\hline Contrastes & Estimativas & Fc & $\mathrm{Pr}>\mathrm{Fc}$ \\
\hline$\overline{\text { Contraste } 1\left(\text { glyphosate }+ \text { chlor }^{1} \mathrm{X} \text { glyphosate }+ \text { carf }^{2}\right)}$ & $-4,97$ & $25,37^{*}$ & 0,0000 \\
\hline Contraste 2 (glyphosate + chlor X glyphosate + chlor + adjuvantes) & $-2,40$ & $1,65^{\mathrm{ns}}$ & 0,2050 \\
\hline Contraste 3 (glyphosate + carf X glyphosate + carf + adjuvantes) & 2,68 & $2,05^{\mathrm{ns}}$ & 0,1580 \\
\hline \multicolumn{4}{|l|}{ Matéria Seca da Parte Aérea } \\
\hline Contraste 1 (testemunha X glyphosate + chlor $^{1}$ e glyphosate + carf $^{2}$ ) & $-3,35$ & $9,78^{*}$ & 0,003 \\
\hline Contraste $2\left(\right.$ glyphosate + chlor $^{1} \mathrm{X}$ glyphosate + carf $\left.^{2}\right)$ & 2,43 & $16,72^{*}$ & 0,0002 \\
\hline Contraste 3 (glyphosate + chlor X glyphosate + chlor + adjuvantes) & 0,19 & $0,03^{\mathrm{ns}}$ & 0,868 \\
\hline Contraste 4 (glyphosate + carf X glyphosate + carf + adjuvantes) & $-2,98$ & $6,98^{*}$ & 0,0109 \\
\hline
\end{tabular}

Obs.: estimativas = estimativa do valor dos contrastes $1 ; 2$ e $3 . \mathrm{Fc}=\mathrm{F}$ calculado; $\mathrm{Pr}>\mathrm{Fc}=$ probabilidade de aceitar ou não a hipótese como verdadeira. ${ }^{1}$ chlorimuron-ethyl; ${ }^{2}$ carfentrazone-ethyl. * significativo a $5 \%$ de probabilidade pelo teste $\mathrm{F} ;{ }^{\text {ns }}$ não significativo pelo teste $\mathrm{F}$, a $5 \%$ de probabilidade.

Os melhores níveis de controle de I. grandifolia foram para glyphosate + carfentrazone-ethyl isolados e associados com Nimbus ${ }^{\circledR}$, Joint Oil ${ }^{\circledR}$, Natur'Oil ${ }^{\circledR}$ e Triunfo $515^{\circledR}$, sendo que, aos 3 DAA, níveis de eficiência foram satisfatórios (> 80\%) (Tabela 6).

$\mathrm{O}$ adjuvante $\mathrm{All} \mathrm{Fix}{ }^{\circledR}$, inicialmente, apresentou eficiência inferior às demais associações com glyphosate + carfentrazone-ethyl, mas aos 21 DAA seu desempenho foi semelhante aos demais tratamentos contendo adjuvantes. Nota-se que o carfentrazone-ethyl, associado com o glyphosate, favorece uma maior velocidade no controle de I. grandifolia, apresentando altas porcentagens de controle com três dias após a aplicação, corroborando da afirmação de ROCHA et al. (2007) de que esta mistura promove interação sinérgica.

CHRISTOFFOLETI et al. (2006) relataram controle satisfatório para I. grandifolia em pósemergência inicial, a partir dos 15 DAA, utilizando carfentrazone-ethyl na dose de $10 \mathrm{~g} \mathrm{ha}^{-1}$. Para a mistura de glyphosate + chlorimuron-ethyl, a adição dos adjuvantes não influenciou no controle de I. grandifolia, mas ainda assim proporcionou nível eficiente $(\geq 88 \%)$ aos 21 DAA.

Esses resultados corroboram os de VIDRINE et al. (2002) e PROCÓPIO et al. (2007), os quais também obtiveram controle satisfatório ( 85 a 90\%) de I. grandifolia 25 e 28 DAA, utilizando a associação de glyphosate + chlorimuron-ethyl nas doses de $700+11 \mathrm{~g} \mathrm{ha}^{-1}$ e $1.440+10 \mathrm{~g} \mathrm{ha}^{-1}$, respectivamente. 
TABELA 6. Controle (\%) aos 3; 7; 14 e 21 DAA e matéria seca da parte aérea de I. grandifolia submetida à aplicação de misturas em tanque, entre glyphosate + chlorimuron-ethyl e glyphosate + carfentrazone-ethyl, isolados e associados a adjuvantes. Control (\%) at 3, 7, 14 and 21 DAA and dossel dry matter of I. grandifolia submitted to tank mixtures application between glyphosate + chlorimuron-ethyl and glyphosate + carfentrazone-ethyl isolated and associated to adjuvants.

\begin{tabular}{|c|c|c|c|c|c|}
\hline Tratamentos & 3 DAA & 7 DAA & 14 DAA & $21 \mathrm{DAA}$ & $\begin{array}{l}\text { Matéria Seca da } \\
\text { Parte Aérea }(\mathrm{g})\end{array}$ \\
\hline 1. gly $^{1}+$ chlor $^{2}$ & $13,0 \mathrm{~d}$ & $34,0 \mathrm{c}$ & $76,0 \mathrm{c}$ & $88,0 \mathrm{~b}$ & $13,17 \mathrm{a}$ \\
\hline 2. gly + chlor + Nimbus ${ }^{\circledR}$ & $19,0 \mathrm{~d}$ & $35,0 \mathrm{c}$ & $75,0 \mathrm{c}$ & $90,6 \mathrm{~b}$ & $12,17 \mathrm{a}$ \\
\hline 3. gly + chlor + Joint Oil $^{\circledR}$ & $16,6 \mathrm{~d}$ & $39,0 \mathrm{c}$ & $82,0 \mathrm{~b}$ & $91,8 \mathrm{~b}$ & $14,24 \mathrm{a}$ \\
\hline 4. gly + chlor + Natur'Oil $^{\circledR}$ & $15,4 \mathrm{~d}$ & $35,8 \mathrm{c}$ & $78,0 \mathrm{c}$ & $90,6 \mathrm{~b}$ & $12,71 \mathrm{a}$ \\
\hline 5. gly + chlor + Triunfo $515^{\circledR}$ & $15,6 \mathrm{~d}$ & $35,0 \mathrm{c}$ & $74,0 \mathrm{c}$ & $90,0 \mathrm{~b}$ & $13,54 \mathrm{a}$ \\
\hline 6. gly + chlor + All Fix ${ }^{\circledR}$ & $13,0 \mathrm{~d}$ & $34,0 \mathrm{c}$ & $75,0 \mathrm{c}$ & $89,0 \mathrm{~b}$ & $11,70 \mathrm{a}$ \\
\hline 7. gly + carfen $^{3}$ & $84,0 \mathrm{~b}$ & 89,0 a & 95,0 a & $97,2 \mathrm{a}$ & $8,10 \mathrm{~b}$ \\
\hline 8. gly + carfen + Nimbus ${ }^{\circledR}$ & $84,0 \mathrm{~b}$ & $90,0 \mathrm{a}$ & $92,6 \mathrm{a}$ & $94,6 \mathrm{a}$ & $13,00 \mathrm{a}$ \\
\hline 9. gly + carfen + Joint Oil ${ }^{\circledR}$ & $86,6 \mathrm{a}$ & $90,0 \mathrm{a}$ & $95,6 \mathrm{a}$ & $96,8 \mathrm{a}$ & $14,38 \mathrm{a}$ \\
\hline 10. gly + carfen + Natur'Oil $^{\circledR}$ & 89,6 a & $90,0 \mathrm{a}$ & $93,2 \mathrm{a}$ & $96,6 \mathrm{a}$ & $7,81 \mathrm{~b}$ \\
\hline 11. gly + carfen + Triunfo $515^{\circledR}$ & $80,0 \mathrm{~b}$ & $85,0 \mathrm{a}$ & $92,0 \mathrm{a}$ & $92,6 \mathrm{~b}$ & $10,49 \mathrm{~b}$ \\
\hline 12. gly + carfen + All Fix ${ }^{\circledR}$ & $42,0 \mathrm{c}$ & $59,0 \mathrm{~b}$ & $80,0 \mathrm{~b}$ & $92,0 \mathrm{~b}$ & $9,70 \mathrm{~b}$ \\
\hline 13. testemunha sem aplicação & $0,0 \mathrm{e}$ & $0,0 \mathrm{~d}$ & $0,0 \mathrm{~d}$ & $0,0 \mathrm{c}$ & $16,46 \mathrm{a}$ \\
\hline$\overline{\mathrm{Pr}}>\mathrm{F}$ & 0,0000 & 0,0000 & 0,0000 & 0,0000 & 0,0000 \\
\hline $\mathrm{Fc}$ & $435,59 *$ & $201,24^{*}$ & $156,86^{*}$ & $228,66^{*}$ & $5,24^{*}$ \\
\hline
\end{tabular}

Obs. : Fc = F calculado; $\mathrm{Pr}>\mathrm{Fc}=$ probabilidade de aceitar ou não a hipotese como verdadeira. ${ }^{1}$ chlorimuron-ethyl; ${ }^{2}$ carfentrazoneethyl; *significativo a 5\% de probabilidade $(\mathrm{p}<0,05)$. Médias seguidas de mesma letra não diferem entre si, a 5\% de probabilidade, pelo teste de Scott-Knot.

Mesmo com altos percentuais de controle de I. grandifolia para todos os tratamentos, destacaram-se com as menores quantidades de matéria seca da parte aérea, aos 21 DAA, os seguintes tratamentos: glyphosate + carfentrazone-ethyl, isolado e associado com Natur' Oil ${ }^{\circledR}$, Triunfo $515^{\circledR}$ e All Fix ${ }^{\circledR}$, ao contrário de glyphosate + carfentrazone-ethyl, associados com Nimbus $^{\circledR}$ e Joint Oil ${ }^{\circledR}$, onde foi observado início de novas brotações, uma vez que as plantas se encontravam próximo à fase de florescimento pleno. Para os demais tratamentos, os valores de acúmulo de matéria seca não diferiram estatisticamente da testemunha, e a justificativa pode ser estabelecida pelo fato de o chlorimuron-ethyl tratar-se de um herbicida sistêmico, de forma semelhante ao glyphosate, os quais normalmente apresentam ação mais lenta no controle das plantas daninhas.

\section{CONCLUSÕES}

Os adjuvantes, apesar de proporcionarem efeitos distintos na redução da tensão superficial e na qualidade da aplicação das misturas em tanque de glyphosate + chlorimuron-ethyl e glyphosate + carfentrazone-ethyl, não interferiram na deposição da calda de pulverização sobre os alvos $I$. grandifolia e lâminas de vidro.

A mistura glyphosate + carfentrazone-ethyl apresentou maior velocidade de controle de $I$. grandifolia em relação ao glyphosate + chlorimuron-ethyl, na ausência ou na presença dos adjuvantes, apesar de o glyphosate + carfentrazone-ethyl, associados com Nimbus ${ }^{\circledR}$ e Joint Oil ${ }^{\circledR}$, terem apresentado início de desenvolvimento de novas folhas.

\section{REFERÊNCIAS}

BANZATTO, D.A.; KRONKA, S.N. Teste de significância. In: BANZATTO, D.A.; KRONKA, S.N. Experimentação agrícola. 4.ed. Jaboticabal: FUNEP, 2008. p.23-52. 
CARVALHO, F.T.; CAVAZZANA, M.A. Eficácia de herbicidas no manejo de plantas daninhas para o plantio direto de soja. Revista Brasileira de Herbicida, Passo Fundo, v. 1, p. 167-172, 2000.

CARVALHO, F.T.; MENDONÇA, M.R.; PERUCHI, M.; PALAZZO, R.R.B. Eficácia de herbicidas no manejo de Euphorbia heterophylla para o plantio direto de soja. Revista Brasileira de Herbicida, Passo Fundo, v. 1, p. 159-165, 2000.

CARVALHO, F.T., PEREIRA, F.A.R.; PERUCHI, M.; PALAZZO, R.R.B. Manejo químico das plantas daninhas Euphorbia heterophylla e Bidens pilosa em sistema de plantio direto da cultura da soja. Planta Daninha, Viçosa-MG, v.21, n.1, p.145-150, 2003.

CHRISTOFFOLETI, P.J.; BORGES, A.; NICOLAI, M.; CARVALHO, S.J.P.; LÓPEZ-OVEJERO, R.F.; MONQUERO, P.A. Carfentrazone-ethyl aplicado em pós-emergência para o controle se Ipomoea spp. e Commelina benghalensis na cultura da cana-de-açúcar. Planta Daninha, ViçosaMG, v.24, n.1, p.83-90, 2006.

DURIGAN, J.C. Efeitos de adjuvantes na aplicação e eficácia de herbicidas. Jaboticabal: FUNEP, 1993. 42p.

EMBRAPA. EMPRESA BRASILEIRA DE PEQUISA AGROPECUÁRIA. Software e-Sprikle. Sadgna.v.1.0. São Carlos, 2000. CD-ROM.

GARCIA, L.C.; RAMOS, H.H.; JUSTINO, A. Avaliação de softwares para análise de parâmetros da pulverização realizada sobre papéis hidrossensíveis. Revista Brasileira de Agrocomputação, Ponta Grossa, v.2, n.1, p.19-28, 2004.

IOST, C.A.R. Efeito de adjuvantes nas propriedades físico-químicas da água e na redução de deriva em pulverizações sobre diferentes espécies de plantas daninhas. 2008. 63 f. Dissertação (Mestrado em Proteção de Plantas) - Faculdade de Ciências Agronômicas, Universidade Estadual Paulista, Botucatu, 2008.

JAREMTCHUCK, C.C.; CONSTANTIN, J.; OLIVEIRA JR., R.S.; BIFFE, D.F.; ALONSO, D.G.; ARANTES, J.G.Z. Efeito de sistemas de manejo sobre a velocidade de dessecação, infestação inicial de plantas daninhas e desenvolvimento e produtividade da soja. Acta Scientiarium Agronomy, Maringá, v.30, n.4, p.449-455, 2008.

KISSMANN, K. G.; GROTH, D. Plantas infestantes e Nocivas. 2.ed. São Paulo: BASF, 1999. $978 \mathrm{p}$.

LACERDA, A.L.S.; VITÓRIA FILHO, R. Curvas dose-resposta em espécies de planas daninhas com o uso do herbicida glyphosate. Bragantia, Campinas, v.63, n.1, p.73-79, 2004.

MACIEL, C.D.G.; VELINI, E.D.; RAETANO, C.G.; SOUZA, R.T.; NEGRISOLI, E.; CAVENAGHI, A.L.; SILVA, M.A.S. Estimativa de depósito e distribuição da calda de pulverização em papel hidrossensível e plantas de Ipomoea grandifolia. In: SIMPÓSIO INTERNACIONAL DE TECNOLOGIA DE APLICAÇÃO DE AGROTÓXICOS, 2., 2001, Jundiaí - SP. Anais... Jundiaí: IAC, 2001.

MACIEL, C.D.G; GUERRA, N.; OLIVEIRA NETO, A.M.; POLETINE, J.P. ; BASTOS, S.L.W.; DIAS, N.M.S. Tensão superficial estática de misturas em tanque de glyphosate + chlorimuron-ethyl isoladas ou associadas com adjuvantes. Planta Daninha, Viçosa-MG, v.28, n.3, p.673-685, 2010.

MACIEL, C.D.G.; VELINI, E.D.; BERNARDO, R.S. Desempenho de pontas de pulverização em Brachiaria brizantha cv. MG-4 para controle de ninfas de cigarrinhas das pastagens. Engenharia Agrícola, Jaboticabal, v.27, n.esp., p.66-74, 2007.

MILLER, P.C.H. Spray drift and its measurements. In: MATTHES, G.A.; HISLOP, E.C. (Ed.). Application technology for crop protection. Trowbridge: CAB international, 1993. p.101-122. 
MONQUERO, P.A.; CHRISTOFFOLETI, P.J.; OSUNA, M.D.; DE PRADO, R.A. Absorção, translocação e metabolismo do glyphosate por plantas tolerantes e sensíveis a este herbicida. Planta Daninha, Viçosa-MG, v.22, n.3, p.445-451, 2004.

MONQUERO, P.A.; CURY, J.C.; CHRISTOFFOLETI, P.J. Controle pelo glyphosate e caracterização geral da superfície foliar de Commelina benghalensis, Ipomoea hederifolia, Richardia brasiliensis e Galinsoga parviflora. Planta Daninha, Viçosa-MG, v.23, n.1, p.123-132, 2005.

PROCÓPIO, S.O.; MENEZES, C.C.E.; BETTA, L.; BETTA, M. Utilização de chlorimuron-ethyl e imazethapyr na cultura da soja roundup ready ${ }^{\circledR}$. Planta Daninha, Viçosa-MG, v.25, n.2, p.365-373, 2007.

ROCHA, D.C.; RODELLA, R.A.; MARTINS, D.; MACIEL, C.D.G. Efeito de herbicidas sobre quatro espécies de trapoeraba. Planta Daninha, Viçosa-MG, v.25, n.2, p.359-364, 2007.

ROMAN, E. S. Eficácia de herbicidas na dessecação e no controle residual de plantas daninhas no sistema desseque e plante. Revista Brasileira de Herbicidas, Passo Fundo, v.3, p.45-49, 2002.

SMITH, D.B. Droplet size and leaf morphology effects on pesticide spray deposition. Transaction $A S A E$, St. Joseph, v.43, p.255-259, 2000.

SBCPD. SOCIEDADE BRASILEIRA DA CIÊNCIA DAS PLANTAS DANINHAS .

Procedimentos para instalação, avaliação e análise de experimentos com herbicidas. Londrina, 1995. $42 \mathrm{p}$.

SCOTT, A.; KNOTT, M. Cluster-analysis method for grouping means in analysis of variance. Biometrics, Raleigh, v.30, n.3, p.507-512, 1974.

STOCK, D.; BRIGGS, G. Physiochemical properties of adjuvants: value and applications. Weed Technology, Champaign, v.14, p.798-806, 2000.

STRAHAN, R.E.; GRIFFIN, J.L.; JORDAN, D.L.; MILLER, D.K. Influence of adjuvants on itchgrass (Rottboellia cochinchinensis) control in corn (Zea mays) with nicosulfuron and primisulfuron. Weed Technology. Champaign, v.14, p.66-71, 2000.

THEISEN, G.; RUEDELL, J.; BIANCHI, M.A. Tecnologia de aplicação de herbicidas: teoria e prática. In: THEISEN, G.; RUEDELL, J. (Ed.). Aspectos técnicos da aplicação de herbicidas. Cruz Alta: Aldeia Norte, 2004, p.25-54.

VANLIESHOUT, L. A.; LOUX, M. M. Interactions of glyphosate with residual herbicides in no-till soybean (Glycine max) production. Weed Technology, Champaign, v.14, p.480-487, 2000.

VIDRINE, P.R.; GRIFFIN, J.L.; BLOUIN, D.C. Evaluation of reduced rates of glyphosate and chlorimuron in glyphosate-resistant soybean. Weed Technology, Champaign, v.16, p.731-736, 2002. 